\title{
Consumer's behavior towards organic food: A case study in Ho Chi Minh City
}

\author{
Hoa T. T. Ha*, Luan D. Tran, \& Linh T. N. Nguyen
}

Faculty of Economics, Nong Lam University Ho Chi Minh City, Ho Chi Minh City, Vietnam

ARTICLE INFO
Research Paper
Received: December 25, 2019
Revised: February 10, 2020
Accepted: March 04, 2020
Keywords
Behavior
Consumer
Intention
Organic food
*Corresponding author
Ha Thi Thu Hoa
Email: hoaha@hcmuaf.edu.vn

Cited as: Ha, H. T. T., Tran, L. D., \& Nguyen, L. T. N. (2020). Consumer's behavior towards organic food: A case study in Ho Chi Minh City. The Journal of Agriculture and Development 19(2), 9-16.

\section{ABSTRACT}

This study aimed to examine factors influencing consumers' behavior towards organic food. Structural equation modelling (SEM) was used to test the relationships between the factors through data collected from 450 consumers in Ho Chi Minh City. Research results showed that consumption intention had the greatest direct impact on consumer behavior. Subjective norms had the strongest influence on consumption intention, followed by the attitude towards organic food. In addition, the price of organic food was a barrier to consumption intention towards this food. At the same time, the results of the multi-group analysis showed that the relationships in the models were not significantly different between male and female, but significantly different among income groups. 


\title{
Hành vi của người tiêu dùng đối với thực phẩm hữu cơ: Trường hợp nghiên cứu ở Thành phố Hồ Chí Minh
}

\author{
Hà Thị Thu Hòa*, Trần Đức Luân \& Nguyễn Thị Nhật Linh \\ Khoa Kinh Tế, Trường Đại Học Nông Lâm TP.HCM, TP. Hồ Chí Minh
}

\section{THÔNG TIN BÀI BÁO \\ Bài báo khoa học \\ Ngày nhận: 25/12/2019 \\ Ngày chỉnh sửa: 10/02/2020 \\ Ngày chấp nhận: 04/03/2020 \\ Từ khóa \\ Hành vi \\ Người tiêu dùng \\ Thực phẩm hữu cơ \\ Ý đinh \\ *Tác giả liên hệ \\ Hà Thị Thu Hòa \\ Email: hoaha@hcmuaf.edu.vn}

\section{TÓM TẮT}

Nghiên cứu này nhằm mục đích xem xét các yếu tố ảnh hưởng đến hành vi của người tiêu dùng đối với thực phẩm hữu cơ (TPHC). Mô hình cấu trúc (SEM) được sử dụng để kiểm định các mối quan hệ thông qua dữ liệu thu thập từ 450 người tiêu dùng trên địa bàn Thành phố Hồ Chí Minh. Kết quả nghiên cứu cho thấy ý định tiêu dùng ảnh hưởng trực tiếp và lớn nhất đến hành vi tiêu dùng. Yếu tố chuẩn chủ quan ảnh hưởng mạnh nhất đến ý định tiêu dùng, tiếp đến là yếu tố thái độ đối với TPHC. Bên cạnh đó, mức giá TPHC là rào cản trong việc thúc đẩy ý định tiêu thụ loại thực phẩm này. Đồng thời, kết quả phân tích đa nhóm cho thấy các mối quan hệ trong mô hình không có sự khác biệt giữa nam và nữ nhưng khác biệt theo các nhóm người tiêu dùng có thu nhập khác nhau.

\section{1. Đặt Vấn Đề}

Thực phẩm là nguồn cung cấp chất dinh dưỡng nuôi sống cơ thể và tạo ra năng lượng cho hoạt động của con người. Tuy nhiên, thực phẩm không an toàn sẽ ảnh hưởng đến sức khỏe, làm giảm sự đáp ứng miễn dịch của cơ thể và có thể gây ung thư. Tiêu chuẩn cuộc sống ngày càng cao cũng như sự quan tâm hơn về sức khỏe, chất lượng và an toàn thực phẩm của người dân đã tạo ra nhu cầu đối với TPHC (Hall \& ctv., 2009; Voon \& ctv., 2011; Wee \& ctv., 2014). Các nghiên cứu trên thế giới phần nào giúp các nhà quản lý hiểu được ý định và hành vi mua TPHC của người tiêu dùng để đưa ra những quyết định marketing thích hợp, đóng góp cho sự phát triển của ngành sản xuất và kinh doanh TPHC (Suh, 2009; Yin \& ctv., 2010; Olivová, 2011). Một số nghiên cứu ở Việt Nam chỉ ra các yếu tố ảnh hưởng đến ý định tiêu dùng thực phẩm an toàn và TPHC như: giá cả, chủng loại hàng, chất lượng, địa điểm bán, thông tin, sự hiểu biết, nhận thức, thái độ và niềm tin khi mua thực phẩm (Huong, 2014; Huong, 2017).

Tiêu dùng TPHC là nhu cầu thiết yếu của người tiêu dùng trong đó có người dân TP.HCM. Tuy nhiên, việc tiêu thụ TPHC ở Việt Nam nói chung và TP.HCM nói riêng vẫn gặp nhiều khó khăn. Từ thực tế đó, việc nghiên cứu hành vi tiêu dùng TPHC của người tiêu dùng TP.HCM trở nên cần thiết. Các nghiên cứu về tiêu dùng TPHC gần đây chỉ phân tích các yếu tố ảnh hưởng đến ý định mua mà chưa tìm hiểu mối quan hệ giữa ý định và hành vi tiêu dùng. Do đó, mục tiêu của nghiên cứu này nhằm tìm hiểu, phân tích ảnh hưởng của các yếu tố đến ý định và hành vi tiêu dùng TPHC. Ngoài ra, nghiên cứu đề xuất giải pháp giúp các cá nhân, đơn vị sản xuất và phân phối, nhà hoạch định chính sách có định hướng phù hợp để khuyến khích tiêu dùng TPHC. 


\section{Cơ Sở Lý Luận và Phương Pháp Nghiên Cứu}

\subsection{Cơ sở lý thuyết}

Có nhiều lý thuyết giải thích cho hành vi mua của người tiêu dùng. Trong đó, hai lý thuyết được sử dụng rộng rãi là Lý thuyết hành vi hợp lý (TRA) (Fishbein \& Ajzen, 1975) và Lý thuyết hành vi có kế hoạch (TPB) (Ajzen, 1991). Lý thuyết TRA cho rằng hành vi của con người là do lý trí kiểm soát hoàn toàn (cá nhân quyết định thực hiện hay không thực hiện hành vi đó). Lý thuyết TPB được mở rộng dựa trên lý thuyết TRA. Trong đó, ý định thực hiện hành vi là mức độ dự định thực hiện hành vi của mỗi người, là sự sẵn sàng để thực hiện hành vi. Như vậy, lý thuyết TPB chỉ ra ba nhân tố độc lập quyết định nên ý định. Thứ nhất là thái độ đối với hành vi (mức độ mà mỗi cá nhân đánh giá cao hay thấp một hành vi nào đó). Thứ hai là chuẩn chủ quan (nhận thức về áp lực xã hội ảnh hưởng đến việc thực hiện hay không thực hiện hành vi). Thứ ba là nhận thức về kiểm soát hành vi (nhận thức về việc dễ hay khó thực hiện một hành vi cụ thể).

Trong nghiên cứu này, thái độ đối với TPHC đóng một vai trò quan trọng trong việc giải thích ý định tiêu dùng. Phần lớn các nghiên cứu thấy mối quan hệ thuận chiều giữa thái độ và ý định tiêu dùng TPHC (Hoyer \& MacInnis, 2007; Dickieson \& ctv., 2009; Chong \& ctv., 2013). Ngoài ra, chuẩn chủ quan là yếu tố dự báo quan trọng về quyết định mua. Những người tham khảo quan trọng càng ủng hộ tích cực thì quyết định mua của người tiêu dùng sẽ thuận lợi hơn (Chen, 2007; Roberts \& Rundle-Thiele, 2007; Tuan, 2011).

Bên cạnh đó, yếu tố sự quan tâm đến sức khỏe, cảm nhận về giá và sự sẵn có là ba thang đo đại diện cho yếu tố kiểm soát hành vi nhận thức trong lý thuyết TPB của Ajzen (1991). Sự quan tâm đến sức khỏe là yếu tố chính thúc đẩy khách hàng mua thực phẩm an toàn (Zanoli \& Naspetti, 2002; Padel \& Foster, 2005). Ngược lại, giá là một trở ngại cho việc mua sản phẩm hữu cơ (Anssi \& Sanna, 2005; Padel \& Foster, 2005). Thêm vào đó, sự sẵn có của sản phẩm luôn xuất hiện như là một nhân tố ủng hộ tích cực ý định mua của người tiêu dùng (Magnusson \& ctv., 2001).

Ý định là đại diện của mặt nhận thức về sự sẵn sàng thực hiện một hành vi, nó được xem như tiền đề đứng trước hành vi. Rezvani \& ctv. (2012) cho rằng ý định là động lực của con người trong chính ý nghĩ thực hiện hành vi. Trong nghiên cứu này, ý định tiêu dùng TPHC được hiểu là ý định của những người đã trải nghiệm việc sử dụng TPHC về việc sẽ tiếp tục sử dụng $\mathrm{TPHC}$ trong thời gian tới.

\subsection{Phương pháp nghiên cứu}

Nghiên cứu chính thức được thực hiện bằng phương pháp nghiên cứu định lượng thông qua phỏng vấn trực tiếp 450 người tiêu dùng ở TP.HCM bằng bảng câu hỏi cấu trúc. Tác giả sử dụng phương pháp chọn mẫu thuận tiện bằng cách đến các cửa hàng, siêu thị trên địa bàn TP.HCM để phỏng vấn trực tiếp người tiêu dùng mua TPHC. Sau quá trình kiểm tra và làm sạch dữ liệu, nghiên cứu tiến hành phân tích thống kê bằng phần mềm SPSS và AMOS. Các phương pháp phân tích số liệu được sử dụng bao gồm thống kê mô tả, phân tích độ tin cậy Cronbach's Alpha, phân tích nhân tố khám phá EFA, phân tích nhân tố khẳng định $\mathrm{CFA}$ và kiểm định mô hình cấu trúc tuyến tính SEM. Căn cứ trên kết quả $\mathrm{CFA}$, mô hình đo lường sẽ được điều chỉnh cho phù hợp với dữ liệu nghiên cứu trước khi thực hiện kiểm định mô hình SEM. Việc đánh giá mức độ phù hợp của mô hình SEM với các chỉ tiêu thống kê Chi-square, CMIN/df, CFI, TLI, GFI và RMSEA. Cuối cùng, kết quả mô hình SEM sẽ được phân tích thông qua các hệ số hồi quy thể hiện ảnh hưởng của các nhóm nhân tố.

Các thang đo sẽ được kiểm định qua hai bước: đánh giá sơ bộ và kiểm định. Nghiên cứu sử dụng thang đo Likert 5 mức độ để đo lường các biến quan sát. Có 7 khái niệm trong nghiên cứu là: thái độ đối với TPHC, chuẩn chủ quan, sự quan tâm đến sức khỏe, cảm nhận về giá, sự sẵn có, ý định và hành vi tiêu dùng TPHC (Bảng 1$)$.

\section{Kết Quả và Thảo Luận}

\subsection{Mẫu nghiên cứu}

Người tiêu dùng được khảo sát chủ yếu là nữ $(86,2 \%)$, điều này cũng phù hợp với thực tế vì nữ giới thường là người nội trợ, mua sắm thực phẩm cho gia đình. Mẫu khảo sát khá đa dạng về tuổi tác, thu nhập cũng như trình độ học vấn. Người tiêu dùng phần lớn thuộc độ tuổi trung niên, từ 25 đến 35 tuổi $(34,4 \%)$ và từ 35 đến 45 tuổi $(31,8 \%)$, đồng thời mức thu nhập chiếm tỷ trọng cao nhất là từ 10 đến 15 triệu (31,8\%). Ngoài ra, học vấn của người tiêu dùng chủ yếu là đại học $(33,8 \%)$, 
Bảng 1. Mô tả các nhân tố

\begin{tabular}{|c|c|c|}
\hline Biến & Mô tả & Nhân tố \\
\hline TD1 & Thực phẩm hữu cơ (TPHC) an toàn hơn thực phẩm thông thường & \multirow{4}{*}{$\begin{array}{c}\text { Thái độ đối } \\
\text { với TPHC } \\
\text { (TD) }\end{array}$} \\
\hline TD2 & Có nhiều loại TPHC để lựa chọn & \\
\hline TD3 & Tôi quan tâm đến các loại TPHC & \\
\hline TD4 & Tôi nghĩ TPHC giàu dinh dưỡng hơn thực phẩm thông thường & \\
\hline CQ1 & Người thân trong gia đình nghĩ rằng tôi nên dùng TPHC & \multirow{4}{*}{$\begin{array}{l}\text { Chuẩn chủ } \\
\text { quan }(\mathrm{CQ})\end{array}$} \\
\hline CQ2 & Bạn bè, dồng nghiệp ủng hộ tôi sử dụng TPHC & \\
\hline CQ3 & Những người quan trọng nhất đối với tôi đang tiêu dùng TPHC & \\
\hline CQ4 & Những người tôi hay tham khảo ý kiến đang tiêu dùng TPHC & \\
\hline SK1 & Sử dụng TPHC là an toàn và tốt hơn cho sức khoẻ & \multirow{4}{*}{$\begin{array}{c}\text { Sự quan tâm } \\
\text { đến sức khỏe } \\
(\mathrm{SK})\end{array}$} \\
\hline SK2 & TPHC chứa các thành phần tự nhiên nên tốt hơn cho sức khỏe & \\
\hline SK3 & Thực phẩm thông thường cũng tốt cho sức khỏe như TPHC & \\
\hline SK4 & $\begin{array}{l}\text { TPHC tốt hơn thực phẩm thông thường vì không/ít chứa thuốc trừ sâu, hóc } \\
\text { môn tăng trưởng và kháng sinh }\end{array}$ & \\
\hline GC1 & Tôi không mua TPHC vì tôi nghĩ chúng đắt tiền & \multirow{4}{*}{$\begin{array}{c}\text { Cảm nhận } \\
\text { về giá (GC) }\end{array}$} \\
\hline GC2 & Giá của TPHC cao hơn thực phẩm thông thường & \\
\hline GC3 & Tôi sẵn sàng chi trả nhiều tiền hơn cho TPHC & \\
\hline GC4 & Một mức giá hợp lí rất quan trọng khi tôi chọn mua TPHC & \\
\hline SC1 & Tôi dễ dàng mua được TPHC vì chúng có sẵn ở các cửa hàng gần nhà & \multirow{4}{*}{$\begin{array}{l}\text { Sự sã̃n có } \\
\quad(\mathrm{SC})\end{array}$} \\
\hline $\mathrm{SC} 2$ & TPHC không dễ dàng tìm thấy ở các cửa hàng gần nhà & \\
\hline $\mathrm{SC} 3$ & Tôi sẽ mua TPHC nếu nó có sẵn tại nơi tôi thường mua sắm & \\
\hline $\mathrm{SC} 4$ & Tôi dễ dàng xác định vị trí các cửa hàng bán TPHC & \\
\hline YD1 & Tôi dự kiến sẽ tăng tỷ lệ TPHC trong tổng lượng thực phẩm tiêu dùng & \multirow{4}{*}{$\begin{array}{c}\text { Ý định tiêu } \\
\text { dùng TPHC } \\
\text { (YD) }\end{array}$} \\
\hline YD2 & Tôi có ý định mua TPHC trong thời gian dài & \\
\hline YD3 & Tôi sẽ tăng tần suất mua TPHC trong thời gian tới & \\
\hline YD4 & Tôi sẽ giới thiệu cho nhiều người khác sử dụng TPHC & \\
\hline HV1 & Tôi thường xuyên mua TPHC & Hành vi tiêu \\
\hline HV2 & Tôi chỉ mua thực phẩm thông thường khi cửa hàng/siêu thị không bán TPHC & dùng TPHC \\
\hline HV3 & Tôi luôn mua TPHC có chứng nhận "hữu cơ" & \\
\hline
\end{tabular}

điều này có thể giúp họ dễ tiếp cận thông tin về TPHC; số lần mua TPHC của người tiêu dùng chủ yếu từ 2 đến 3 lần mỗi tuần.

\subsection{Kiểm định thang đo}

Trong phần kiểm định độ tin cậy thang đo các biến độc lập và phụ thuộc có Cronbach's Alpha $>0,6$, nên phù hợp để sử dụng trong mô hình và được đưa vào phân tích nhân tố EFA. Khi phân tích EFA, có một thang đo thuộc biến Cảm nhận về giá bị loại là GC5. Kết quả phân tích EFA của các thang đo Thái độ đối với TPHC (TD); Chuẩn chủ quan (CQ); Sự quan tâm đến sức khỏe (SK); Cảm nhận về giá (GC); Sự sẵn có (SC); Ý định tiêu dùng TPHC (YD) và Hành vi tiêu dùng TPHC $(\mathrm{HV})$ có hệ số $\mathrm{KMO}=0,909>0,5$, Eigenvalue $=1,002$, phương sai trích $=68,514 \%$ và kiểm định Barlett's có mức ý nghĩa sig $=0,000$
$<0,05$ nên có thể xem phân tích nhân tố là phù hợp (Anderson \& Gerbing, 1988). Cho phép kết luận không có hiện tượng tương quan giữa các biến.

Để khẳng định độ tin cậy và giá trị thang đo, phương pháp CFA được áp dụng với phương pháp ước lượng Maximum Likelihood. Mô hình này có Chi-square $=551,589 ;$ df $=303(P=0,000)$; $\mathrm{CMIN} / \mathrm{df}=1,820(<3)$, đạt được yêu cầu cho độ tương thích. Các hệ số tải nhân tố (Bảng 2) đều $>0,5$ và có ý nghĩa thống kê. Do đó các biến quan sát có liên hệ chặt chẽ với nhân tố đại diện của chúng. Hơn nữa, các chỉ tiêu khác cũng đạt được yêu cầu (TLI $=0,965 ; \mathrm{CFI}=0,970$, GFI $=0,917$; và $R M S E A=0,043(<0,08))$. Vì vậy có thể kết luận mô hình phù hợp với dữ liệu thị trường (Tho \& Trang, 2011). Kết quả CFA khẳng định tính đơn hướng (do sự phù hợp của mô hình và không có tương quan giữa các sai số của các 
Bảng 2. Hệ số tải nhân tố đã chuẩn hóa

\begin{tabular}{|c|c|c|c|c|c|c|c|}
\hline Biến $^{1}$ & TD & $\mathrm{CQ}$ & SK & $\mathrm{GC}$ & $\mathrm{SC}$ & $\mathrm{YD}$ & $\mathrm{HV}$ \\
\hline TD1 & 0,774 & & & & & & \\
\hline TD2 & 0,883 & & & & & & \\
\hline TD3 & 0,860 & & & & & & \\
\hline TD4 & 0,881 & & & & & & \\
\hline CQ1 & & 0,794 & & & & & \\
\hline CQ2 & & 0,832 & & & & & \\
\hline CQ3 & & 0,808 & & & & & \\
\hline CQ4 & & 0,843 & & & & & \\
\hline SK1 & & & 0,893 & & & & \\
\hline SK2 & & & 0,877 & & & & \\
\hline SK3 & & & 0,804 & & & & \\
\hline SK4 & & & 0,917 & & & & \\
\hline GC1 & & & & 0,735 & & & \\
\hline GC2 & & & & 0,742 & & & \\
\hline GC3 & & & & 0,701 & & & \\
\hline GC4 & & & & 0,707 & & & \\
\hline SC1 & & & & & 0,832 & & \\
\hline $\mathrm{SC} 2$ & & & & & 0,873 & & \\
\hline $\mathrm{SC} 3$ & & & & & 0,904 & & \\
\hline $\mathrm{SC} 4$ & & & & & 0,866 & & \\
\hline YD1 & & & & & & 0,705 & \\
\hline YD2 & & & & & & 0,868 & \\
\hline YD3 & & & & & & 0,804 & \\
\hline YD4 & & & & & & 0,762 & \\
\hline HV1 & & & & & & & 0,832 \\
\hline HV2 & & & & & & & 0,852 \\
\hline HV 3 & & & & & & & 0,795 \\
\hline
\end{tabular}

${ }^{1}$ TD: Thài độ; CQ: chủ quan; SK: sức khoẻ; GC: cảm nhận về giá; $\mathrm{SC}$ : sự sẵn có; YD: ý định; HV: hành vi.

Bảng 3. Kết quả kiểm định thang đo các khái niệm đơn hướng

\begin{tabular}{lccccc}
\hline Thành phần & $\begin{array}{c}\text { Số biến } \\
\text { quan sát }\end{array}$ & $\begin{array}{c}\text { Độ tin } \\
\text { cậy tổng } \\
\text { hợp (CR) }\end{array}$ & $\begin{array}{c}\text { Tổng } \\
\text { phương sai } \\
\text { trích (AVE) }\end{array}$ & $\begin{array}{c}\text { Trung } \\
\text { bình hệ } \\
\text { số tải }\end{array}$ & $\begin{array}{c}\text { Giá trị hội } \\
\text { tụ và phân } \\
\text { biệt }\end{array}$ \\
\hline $\begin{array}{l}\text { Thái độ đối với thực phẩm } \\
\text { hữu cơ (TPHC) }\end{array}$ & 4 & 0,913 & 0,724 & 0,850 & Thỏa mãn \\
$\begin{array}{l}\text { Chuẩn chủ quan } \\
\text { Sự quan tâm đến sức khỏe }\end{array}$ & 4 & 0,891 & 0,672 & 0,819 & Thỏa mãn \\
Cảm nhận về giá & 4 & 0,928 & 0,763 & 0,873 & Thỏa mãn \\
Sự sã̃n có & 4 & 0,813 & 0,521 & 0,721 & Thỏa mãn \\
Ý định tiêu dùng TPHC & 4 & 0,925 & 0,755 & 0,869 & Thỏa mãn \\
Hành vi tiêu dùng TPHC & 3 & 0,866 & 0,619 & 0,785 & Thỏa mãn \\
\hline
\end{tabular}

biến quan sát) và giá trị hội tụ của 7 thang đo vì các trọng số $\left(\lambda_{\mathrm{i}}\right)$ đều khá cao (thấp nhất là YD1 $=0,755)$ và có ý nghĩa thống kê (các giá trị $P$ đều bé hơn 0,05$)$. Như vậy, các biến quan sát dùng để đo lường các khái niệm đơn hướng đều đạt giá trị hội tụ (Anderson \& Gerbing, 1988).
Ngoài ra, độ tin cậy tổng hợp thang đo các khái niệm đơn hướng đều đạt giá trị $>0,7$ (thấp nhất là 0,813 ) và tổng phương sai trích $>0,5$ (thấp nhất là 0,521 ) (Bảng 3). Do đó, các thang đo của các khái niệm đơn hướng đạt yêu cầu về giá trị và độ tin cậy. Kết quả kiểm định thang đo các 


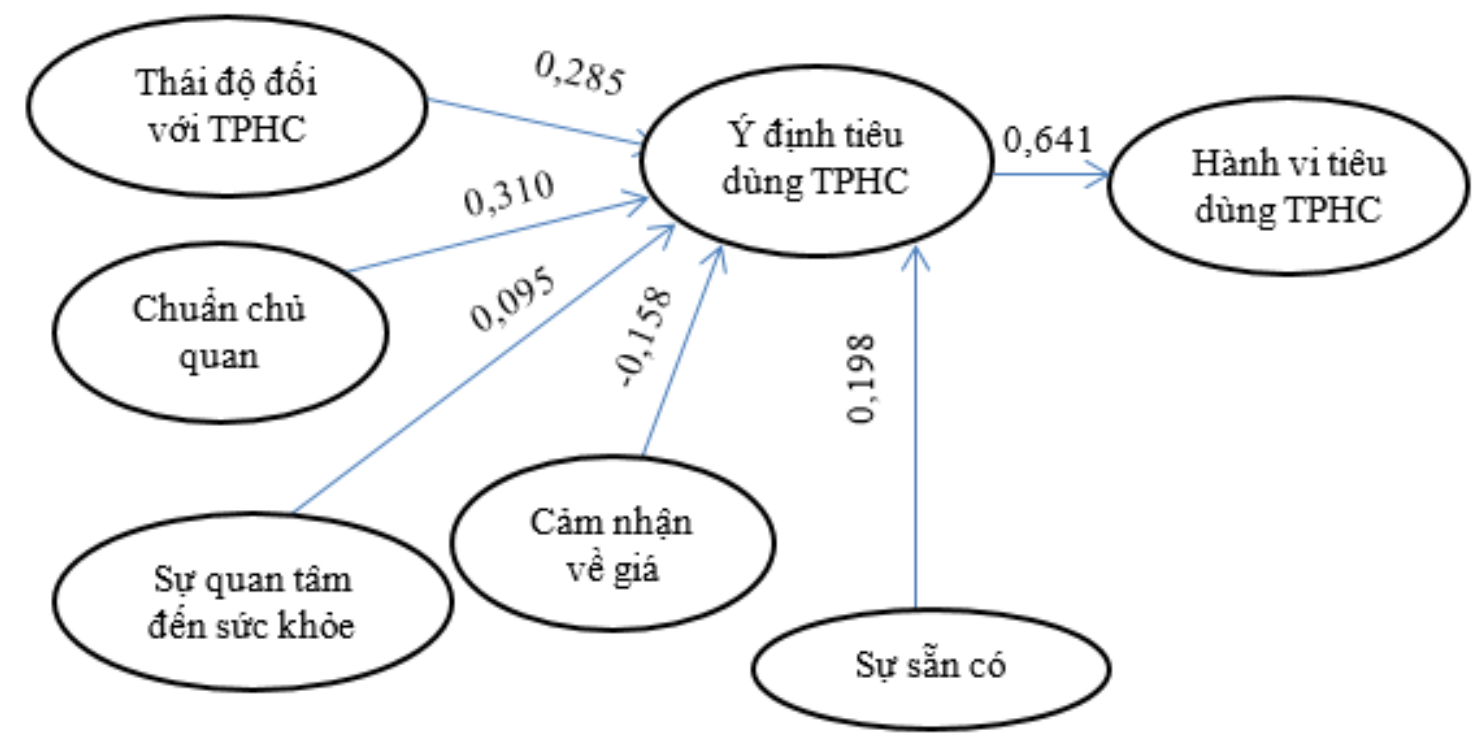

Hình 1. Mô hình cấu trúc hành vi tiêu dùng thực phẩm hữu cơ (TPHC).

khái niệm đơn hướng

Ngoài ra, các hệ số tương quan giữa các khái niệm nghiên cứu nhỏ hơn 1 , có ý nghĩa thống kê $(P=0,000<0,05)$ và các hệ số tới hạn C.R $>$ 2. Hay nói cách khác, các khái niệm nghiên cứu đạt giá trị phân biệt.

\subsection{Kiểm định mô hình nghiên cứu và các giả thuyết}

Phân tích SEM được thực hiện với các chỉ tiêu đo lường độ phù hợp của mô hình với dữ liệu tương tự CFA. Kết quả phân tích SEM của mô hình có $\mathrm{df}=308$, Chi-square là 659,501 với $P=$ 0,000 . Với $\mathrm{CMIN} / \mathrm{df}=2,141<3$ và các chỉ số đánh giá mức độ phù hợp đều đạt yêu cầu (TLI $=0,951 ; \mathrm{CFI}=0,957 ; \mathrm{GFI}=0,903 ; \mathrm{RMSEA}=$ 0,050). Như vậy có thể kết luận mô hình thích hợp với dữ liệu thị trường.

Kết quả kiểm định SEM thể hiện yếu tố ý định tiêu dùng ảnh hưởng đến hành vi tiêu dùng với hệ số lớn nhất $(0,641)$. Ngoài ra, hành vi tiêu dùng còn chịu ảnh hưởng gián tiếp bởi các yếu tố khác như Chuẩn chủ quan, Sự quan tâm đến sức khỏe, Cảm nhận về giá, Sự sẵn có (thông qua ý định tiêu dùng). Trong đó, yếu tố chuẩn chủ quan tác động mạnh nhất đến ý định tiêu dùng $(0,310)$, tiếp đến là thái độ $(0,285)$, sự sã̃n có $(0,198)$, giá cả $(-0,158)$ và thấp nhất là sự quan tâm đến sức khỏe $(0,095)$. Trong tất cả các nhân tố, giá cả là nhân tố ảnh hưởng ngược chiều đến ý định tiêu dùng (Hình 1). Các kết quả này có thể được lý giải rõ hơn trong thực tế. Khi những người xung quanh (gia đình, người thân, đồng nghiệp, bạn bè, hàng xóm) đều cho rằng nên sử dụng TPHC, điều này thúc đẩy người tiêu dùng sẽ lựa chọn thực phẩm này. Đồng thời, người tiêu dùng càng nhận thức lợi ích của TPHC thì càng có thái độ tích cực và thúc đẩy ý định tiêu dùng. Người tiêu dùng dễ tìm thấy TPHC gần nơi sinh sống sẽ gia tăng ý định tiêu dùng. Ngoài ra, hiện nay vấn đề thực phẩm không an toàn đang báo động, đồng thời đời sống người dân được cải thiện nên họ rất quan tâm đến sức khỏe. Vì vậy người tiêu dùng sẽ chọn TPHC cho bản thân và gia đình. Tuy nhiên hiện nay giá TPHC khá cao (do chi phí sản xuất, phí chứng nhận, quản lý) nên nhiều người tiêu dùng vẫn chưa lựa chọn loại TPHC này.

Ngoài ra, các hệ số đường dẫn trong mô hình SEM đều có ý nghĩa thống kê $(P<0,05)$ và phù hợp với mô hình (Bảng 4). Do đó, các giả thuyết $\mathrm{H} 1, \mathrm{H} 2, \mathrm{H} 3, \mathrm{H} 4, \mathrm{H} 5$ và $\mathrm{H} 6$ đều được chấp nhận. Kết quả kiểm định các giả thuyết khẳng định mối quan hệ có ý nghĩa thống kê giữa các yếu tố trong mô hình.

Hệ số $\mathrm{R}^{2}$ cho thấy $41 \%$ sự thay đổi hành vi tiêu dùng TPHC của người tiêu dùng trên địa bàn TP.HCM được giải thích bởi nhân tố ý định tiêu dùng. Và $54,6 \%$ sự biến thiên của ý định tiêu dùng được giải thích bởi năm yếu tố: Thái độ đối 
Bảng 4. Quan hệ giữa các khái niệm trong mô hình

\begin{tabular}{ccccccc}
\hline Giả thuyết & Mối quan hệ $^{1}$ & Estimate & S.E. & C.R. & $P$ & Kiểm định \\
\hline H1 & TD $\longrightarrow$ YD & 0,300 & 0,051 & 5,890 & 0,000 & Chấp nhận \\
H2 & CQ $\longrightarrow$ YD & 0,330 & 0,057 & 5,826 & 0,000 & Chấp nhận \\
H3 & SK $\longrightarrow$ YD & 0,099 & 0,050 & 1,975 & 0,048 & Chấp nhận \\
H4 & GC $\longrightarrow$ YD & $-0,165$ & 0,050 & $-3,285$ & 0,001 & Châ̂p nhận \\
H5 & SC $\longrightarrow$ YD & 0,195 & 0,043 & 4,499 & 0,000 & Châ̂p nhận \\
H6 & YD $\longrightarrow$ HV & 0,546 & 0,043 & 12,605 & 0,000 & Châ̂p nhận \\
\hline
\end{tabular}

${ }^{1}$ TD: Thái độ đối với thực phẩm hữu cơ; $\mathrm{CQ}$ : Chuẩn chủ quan; SK: Sự quan tâm đến sức khỏe; GC: Cảm nhận về giá; SC: Sự sã̃n có; YD: Ý định tiêu dùng thực phẩm hữu cơ; HV: Hành vi tiêu dùng thực phẩm hữu cơ.

Bảng 5. Kết quả kiểm định mô hình bất biến từng phần và khả biến

\begin{tabular}{clccc}
\hline Biến kiểm định & Mô hình so sánh & Chi-square & Df & $P$ \\
\hline \multirow{3}{*}{ Giới tính } & Bất biến từng phần & 1105,807 & 622 & 0,000 \\
& Khả biến & 1096,752 & 616 & 0,000 \\
& Giá trị khác biệt & 9,055 & 6 & 0,171 \\
\hline \multirow{3}{*}{ Thu nhập } & Bất biến từng phần & 1081,805 & 622 & 0,000 \\
& Khả biến & 1059,654 & 616 & 0,000 \\
& Giá trị khác biệt & 22,151 & 6 & 0,011 \\
\hline
\end{tabular}

với TPHC, Chuẩn chủ quan, Sự quan tâm đến sức khỏe, Cảm nhận về giá và Sự sẵn có.

Nghiên cứu sử dụng Bootstrap với số lượng mẫu lặp lại $\mathrm{N}=1000$ để kiểm định độ tin cậy của các ước lượng (Schumacker \& Lomax, 1996). Kết quả cho thấy độ lệch rất bé $(P<0,05)$ nên có thể kết luận các ước lượng trong mô hình là tin cậy được.

Nghiên cứu còn tiến hành phân tích đa nhóm theo biến giới tính và thu nhập (Bảng 5 ). Kết quả cho thấy mô hình khả biến và bất biến từng phần không khác biệt theo hai nhóm giới tính (nam hay nữ thì cả 5 nhân tố đều ảnh hưởng đến YD - mô hình bất biến được chọn) nhưng có sự khác biệt theo các nhóm thu nhập (nhân tố CQ, GC có ý nghĩa với nhóm thu nhập thấp nhưng không có ý nghĩa với nhóm thu nhập cao, còn nhân tố SC thì ngược lại - mô hình khả biến được chọn).

\section{Kết Luận}

Các yếu tố: Thái độ đối với TPHC, Chuẩn chủ quan, Sự quan tâm đến sức khỏe, Cảm nhận về giá và Sự sã̃n có ảnh hưởng đến Ý định và Hành vi tiêu dùng TPHC. Mô hình SEM chứng minh Ý định tiêu dùng là đại diện cho sự sẵn sàng thực hiện một hành vi, có sự ảnh hưởng trực tiếp và lớn nhất đến Hành vi tiêu dùng. Bên cạnh đó, mức giá TPHC hiện tại cao hơn nhiều với các loại thực phẩm thông thường là rào cản trong việc thúc đẩy tiêu thụ. Ngoài ra, nghiên cứu còn cho thấy mối quan hệ giữa các yếu tố trong mô hình không có sự khác biệt theo giới tính nhưng có khác biệt theo các nhóm thu nhập.

Mặc dù nghiên cứu này xác định được các yếu tố quan trọng ảnh hưởng đến hành vi tiêu dùng TPHC, đo lường được mối quan hệ giữa ý định và hành vi tiêu dùng TPHC nhưng còn nhiều yếu tố khác như niềm tin, truyền thông, chất lượng, kiến thức TPHC và sự quan tâm đến môi trường chưa được đo lường. Khi đo lường mối quan hệ giữa các biến, nghiên cứu còn bỏ qua đặc điểm cá nhân, tác động marketing và môi trường marketing. Những hạn chế này cần được tiến hành khảo sát và tiếp tục nghiên cứu thêm.

\section{Tài Liệu Tham Khảo (References)}

Ajzen, I. (1991). The theory of planned behavior. Organizational behavior and human decision processes $50(2)$, 179-211.

Anderson, J. C., \& Gerbing, D. W. (1988). Structural equation modeling in practice: A review and recommended two-step approach. Psychological bulletin 103(3), 411-423.

Anssi, T., \& Sanna, S. (2005). Subjective norms, attitudes and intentions of Finnish consumers in buying organic food. British Food Journal 107(11), 808-822.

Chen, M. F. (2007). Consumer attitudes and purchase intentions in relation to organic foods in Taiwan: Mod- 
erating effects of food-related personality traits. Food Quality and Preference 18(7), 1008-1021.

Chong, C. W., Nahid, N., \& Shamsollahi, A. (2013). Factors influencing on purchasing behaviour of organic foods. Human and Social Science Research 1(2), 93104.

Dickieson, J., Arkus, V., \& Wiertz, C. (2009). Factors that influence the purchase of organic food: A study of consumer behaviour in the UK. Cass Business School, London, England.

Fishbein, M., \& Ajzen, I. (1975). Belief, attitude, intention, and behavior: An introduction to theory and research. Boston, USA: Addison-Wesley.

Hall, J. N., Moore, S., Harper, S. B., \& Lynch, J. W. (2009). Global variability in fruit and vegetable consumption. American Journal of Preventive Medicine 36(5), 402-409.

Hoyer, W. D., \& MacInnis, D. J. (2007). Consumer behavior ( $4^{\text {th }}$ ed.). Boston, America: Houghton Mifflin Co.

Huong, T. L. (2014). Factors influencing urban residents' intention to buy safe food in Ha Noi city (Unpublished doctoral dissertation). National Economics University, Ha Noi, Vietnam.

Huong, T. T. N. (2017). Purchasing behaviors toward safe foods and applying them to marketing activities at the retail business of food products in Ha Noi (Unpublished doctoral dissertation). University of Commerce, Ha Noi, Vietnam.

Magnusson, M. K., Arvola, A., Hursti, U. K. K., Aberg, L., \& Sjoden, P. O. (2001). Attitudes towards organic foods among Swedish consumers. British Food Journal 103(3), 209-227.

Olivová, K. (2011). Intention to buy organic food among consumers in the Czech Republic (Master's thesis). University of Agder, Kristiansand, Norway.

Padel, S., \& Foster, C. (2005). Exploring the gap between attitudes and behaviour: Understanding why consumers buy or do not buy organic food. British Food Journal 107(8), 606-625.

Rezvani, S., Dehkordi, G. J., Rahman, M. S., Fouladivanda, F., Habibi, M., \& Eghtebasi, S. (2012). A conceptual study on the country of origin effect on consumer purchase intention. Asian Social Science 8(12), 205-215.
Roberts, R., \& Rundle-Thiele, S. R. (2007). Organic food: observations of Chinese purchasing behaviors. Proceedings of the 2007 Australian and New Zealand Marketing Academy Conference (ANZMAC 2007) (34303436). Dunedin, New Zealand: University of Otago, School of Business.

Schumacker, E. R., \& Lomax, G. R. (1996). A beginner's guide to structural equation modeling. New Jersey, America: Erlbaum.

Suh, B. W. (2009). Factors influencing consumers' perceptions, intention to purchase and realised purchase behaviour for organic food in South Korea (Unpublished doctoral dissertation). University of Surrey, Surrey, UK.

Tho, D. N., \& Trang, T. M. N. (2011). Scientific research marketing: SEM linear structure model application ( $2^{\text {nd }}$ ed.). Ho Chi Minh City, Vietnam: HCMC National University Publisher.

Tuan, P. N. (2011). A comparative study of the intention to buy organic food between consumers in Northern and Southern Vietnam. AU-GSB e-Journal 4(2), 102113.

Voon, J. P., Ngui, K. S., \& Agrawal, A. (2011). Determinants of willingness to purchase organic food: An exploratory study using structural equation modeling. International Food and Agribusiness Management Review 14(2), 103-120.

Wee, C. S., Ariff, M. S. B. M., Zakuan, N., Tajudin, M. N. M., Ismail, K., \& Ishak, N. (2014). Consumers perception, purchase intention and actual purchase behavior of organic food products. Review of Integrative Business and Economics Research 3(2), 378-397.

Yin, S., Wu, L., Du, L., \& Chen, M. (2010). Consumers' purchase intention of organic food in China. Journal of the Science of Food and Agriculture 90(8), 1361-1367.

Zanoli, R., \& Naspetti, S. (2002). Consumer motivations in the purchase of organic food: a means-end approach. British Food Journal 104(8), 643-653. 